\title{
Optical diagnosis of colorectal lesions requires technology, dedication, and knowledge of its limits
}

Authors

Institutions
Ana Ignjatovic Wilson ${ }^{1}$, Tonya Kaltenbach ${ }^{2}$

${ }^{1}$ Wolfson Unit for Endoscopy, St Mark's Hospital, Imperial College, London, United Kingdom

${ }^{2}$ Veterans Affairs Palo Alto, Stanford University School of Medicine, Palo Alto, California, United States submitted 27. August 2014 accepted after revision: 8. September 2014

Bibliography Dol http://dx.doi.org/ 10.1055/s-0034-1390710 Endoscopy International Open 2014; 02: E133-E134 (c) Georg Thieme Verlag KG Stuttgart · New York E-ISSN 2196-9736

Corresponding author Ana Wilson

St Mark's Hospital, Imperial College, London, UK Fax: +44 2084233588 ana.wilson@nhs.net

\section{Abbreviations \\ $\nabla$ \\ FIT fecal immunochemical testing \\ NBI narrow band imaging \\ NICE NBI International Colorectal Endoscopic \\ PIVI Preservation and Incorporation of Valu- able Endoscopic Innovations}

Optical diagnosis of small colorectal polyps without the need for histopathology has the potential to improve the cost effectiveness of colonoscopy by reducing the time for polyp retrieval and the cost of histopathology [1,2]. In addition, the ability to tell patients the surveillance interval needed immediately after the procedure reduces the cost associated with follow-up and alleviates patient anxiety. Many studies, including several meta-analyses [3 -5], have shown that optical diagnosis of small colorectal polyps is safe and feasible in routine clinical practice and that the results are comparable to those found using histopathology, the current reference standard. In academic centers and in vivo settings, experienced endoscopists have achieved 93\% concordance of surveillance intervals needed when made by optical diagnosis and histopathology and $>90 \%$ negative predictive value for rectosigmoid polyps.

Because the risk of malignancy increases with the size of the polyp ( $>1 \mathrm{~cm}$ ), most studies have focused on evaluating the accuracy of optical diagnosis of smaller polyps $(<10 \mathrm{~mm})$. Americans, however, are uncomfortable with even the small risk of advanced malignancy that $6-9 \mathrm{~mm}$ polyps may harbor and tend to concentrate on diagnosis of diminutive polyps ( $<6 \mathrm{~mm}$ ). The American Society of Gastrointestinal Endoscopy Preservation and Incorporation of Valuable Endoscopic Innovations established diagnostic thresholds for real time endoscopic assessment of the histology of diminutive colorectal polyps to facilitate standardized research and implementation in clinical practice [6].
There are two proposed thresholds for optical diagnosis of diminutive colorectal polyps:

1. For colorectal polyps $\leq 5 \mathrm{~mm}$ to be resected and discarded without histopathologic assessment, endoscopic technology (used with high confidence) to determine histology combined with histopathologic assessment should provide $\mathrm{a} \geq$ $90 \%$ agreement in the assignment of post-polypectomy surveillance intervals when compared to decisions based on pathology assessment of identified polyps.

2. For a technology to be used to guide the decision to leave suspected rectosigmoid hyperplastic polyps $\leq 5 \mathrm{~mm}$ in place (without resection), the technology (used with high confidence) should provide $\geq 90 \%$ negative predictive value for adenomatous histology.

The study by Stegeman et al published in this Issue failed to fulfill the second criterion (the first criterion was not assessed) and the authors concluded that the accuracy of optical diagnosis for colonic lesions is not acceptable for colonoscopies for patients with positive fecal immunochemical testing (FIT) results. Their study highlights many issues with the studies of optical diagnosis and the conclusions drawn.

\section{Defining optical diagnosis}

In the context in which optical diagnosis of colorectal polyps is used as a basis for deciding to discard polyps or leave hyperplastic polyps in situ, the initial Detect InSpect ChAracterise Resect and Discard (DISCARD) study included only polyps smaller than $10 \mathrm{~mm}$ because the risk for advanced neoplasia increases significantly in bigger lesions [2]. Since the publication of Preservation and Incorporation of Valuable Endoscopic Innovations (PIVI) there is agreement among experts that only lesions smaller than $6 \mathrm{~mm}$ should be included in studies of optical diagnosis because it is in those lesions that the risk of advanced neoplasia 
is small. Indeed, this was confirmed by Stegeman et al. where all cancers detected were $>10 \mathrm{~mm}$.

\section{Defining outcomes}

From the PIVI recommendations, the outcomes should include both the accuracy of optical diagnosis (in particular, the negative predictive value for adenomatous histology) and, of more relevance to the patient, concordance of surveillance intervals as determined by optical diagnosis and histopathology.

\section{Using appropriate technology}

There are many studies in the literature that have convincingly shown that white light alone is not sufficient for optical diagnosis because its accuracy is significantly lower than the accuracy of histopathology [7-10]. Studies that have shown white light results that are comparable to histopathology results have all used technology in addition to white light. Chromoendoscopy and more recently narrow spectra technologies such as narrow band imaging (NBI; Olympus) have been most often studied. Stegeman et al offered no clear protocol for the use of technologies in addition to white light and hence it is unclear whether the results represent the use of white light alone or an unknown combination of white light and other add-on techniques.

\section{Standardized criteria}

To date the NBI International Colorectal Endoscopic (NICE) classification [11] is the only validated criterion for the classification of neoplastic and non-neoplastic polyps as well those with deep submucosal invasion [12] when using NBI during real time colonoscopy. Other classification systems for NBI, other narrow spectra technologies, and chromoendoscopy have been described. In addition, the level of confidence in the results should always be considered when making an optical diagnosis-the endoscopist must be confident of the diagnosis before lesions are resected and discarded or left in situ. When the endoscopist has low confidence in the optical diagnosis, polyps should be sent for histopathology.

\section{Training in optical diagnosis}

Much like performing colonoscopy, optical diagnosis requires training. We developed a PowerPoint training module using still images (with validated construct and content), which demonstrated that a short training session can improve the accuracy of optical diagnosis [13]. Rastogi et al [14] showed similar results using video images and, in addition to improved diagnostic accuracy after training, revealed an increase in the proportion of high confidence diagnoses. There are many other training modules available and studies assessing optical diagnosis should use these validated tools, and only those endoscopists who 'pass' the training should participate in the in vivo part of the study. In addition, endoscopists should receive active feedback on their optical diagnoses and assessment when the study is underway to ensure sustained performance.

\section{Photo documentation}

The ability to take a clear image of the polyp on which optical diagnosis is made is of paramount importance because it serves as a record for both the patient and the self-audit for the endoscopist. Optimization of the quality of the image contributes to consistent and reliable image capture.

To conclude, optical diagnosis of small colorectal polyps was envisaged to be used for selected lesions-those without suspicious features that would suggest advanced neoplasia (eg, irregular shape, central depression, irregular color) when assessed using white light endoscopy and in which endoscopists were confident of their diagnosis using white light endoscopy with additional technology (chromoendoscopy/narrow spectra technologies). Optical diagnosis was never meant to replace histopathology but was an attempt to reduce the workload in assessing diminutive polyps, which are increasingly being detected, with improved standards of colonoscopy. The study design offered by Stegeman et al does not effectively address whether the technique for optical diagnosis of diminutive colorectal polyps could be translated into routine clinical practice. Thus, the reasons for the poor performance of optical diagnosis of polyps found are not necessarily due to an inherent inaccuracy in the technique, but are caused by its incorrect application.

\section{Competing interests: None}

\section{References}

1 Hassan C, Pickhardt PJ, Rex DK. A resect and discard strategy would improve cost-effectiveness of colorectal cancer screening. Clin Gastroenterol Hepatol 2010; 8: 865-869 [869 e1-e3]

2 Ignjatovic A, East JE, Suzuki $N$ et al. Optical diagnosis of small colorectal polyps at routine colonoscopy (Detect InSpect ChAracterise Resect and Discard; DISCARD trial): a prospective cohort study. Lancet Oncol 2009; 10: $1171-1178$

3 McGill SK, Evangelou E, Ioannidis JP et al. Narrow band imaging to differentiate neoplastic and non-neoplastic colorectal polyps in real time: a meta-analysis of diagnostic operating characteristics. Gut 2013; 62: $1704-1713$

$4 \mathrm{Wu} L, \mathrm{Li} Y, \mathrm{Li} Z$ et al. Diagnostic accuracy of narrow-band imaging for the differentiation of neoplastic from non-neoplastic colorectal polyps: a meta-analysis. Colorectal Dis 2013; 15: 3-11

5 Wanders LK, East JE, Uitentuis SE et al. Diagnostic performance of narrowed spectrum endoscopy, autofluorescence imaging, and confocal laser endomicroscopy for optical diagnosis of colonic polyps: a metaanalysis. Lancet Oncol 2013; 14: 1337-1347

6 Rex DK, Kahi C, O'Brien $M$ et al. The American Society for Gastrointestinal Endoscopy PIVI (Preservation and Incorporation of Valuable Endoscopic Innovations) on real-time endoscopic assessment of the histology of diminutive colorectal polyps. Gastrointest Endosc 2011; 73: 419-422

7 Apel D, Jakobs R, Schilling $D$ et al. Accuracy of high resolution of chromoendoscopy in prediction of histologic findings in diminutive lesions of the rectosigmoid. Gastrointest Endosc 2006; 63: 824-828

8 Machida H, Sano Y, Hamamoto Y et al. Narrow-band imaging in the diagnosis of colorectal mucosal lesions: a pilot study. Endoscopy 2004; 36: $1094-1098$

9 Fu KI, Sano Y, Kato $S$ et al. Chromoendoscopy using indigo carmine dye spraying with magnifying observation is the most reliable method for differential diagnosis between non-neoplastic and neoplastic colorectal lesions: a prospective study. Endoscopy 2004; 36: 1089-1093

10 Tischendorf JJ, Wasmuth HE, Koch A et al. Value of magnifying chromoendoscopy and narrow band imaging (NBI) in classifying colorectal polyps: a prospective controlled study. Endoscopy 2007; 39: $1092-$ 1096

11 Hewett DG, Kaltenbach T, Sano Y et al. Validation of a simple classification system for endoscopic diagnosis of small colorectal polyps using narrow-band imaging. Gastroenterology 2012; 143: 599-607 e1

12 Hayashi $N$, Tanaka S, Hewett DG et al. Endoscopic prediction of deep submucosal invasive carcinoma: validation of the narrow-band imaging international colorectal endoscopic (NICE) classification. Gastrointest Endosc 2013; 78: 625-632

13 Ignjatovic A, Thomas-Gibson S, East JE et al. Development and validation of a training module on the use of narrow-band imaging in differentiation of small adenomas from hyperplastic colorectal polyps. Gastrointest Endosc 2011; 73: 128 - 133

14 Rastogi A, Rao DS, Gupta $N$ et al. Impact of a computer-based teaching module on characterization of diminutive colon polyps by using narrow-band imaging by non-experts in academic and community practice: a video-based study. Gastrointest Endosc 2014; 79: 390-398 